\title{
WiFi Positioning Based on User Orientation Estimation and Smartphone Carrying Position Recognition
}

\author{
Zhi-An Deng $\mathbb{D}^{D}$, Zhiyu Qu $\mathbb{D}^{\text {D }}$, Changbo Hou, Weijian Si, and Chunjie Zhang \\ College of Information and Communication Engineering, Harbin Engineering University, Harbin 150001, China \\ Correspondence should be addressed to Zhiyu Qu; quzhiyu@hrbeu.edu.cn
}

Received 28 December 2017; Revised 24 March 2018; Accepted 15 April 2018; Published 27 September 2018

Academic Editor: Xin-Lin Huang

Copyright (C) 2018 Zhi-An Deng et al. This is an open access article distributed under the Creative Commons Attribution License, which permits unrestricted use, distribution, and reproduction in any medium, provided the original work is properly cited.

\begin{abstract}
Accuracy performance of WiFi fingerprinting positioning systems deteriorates severely when signal attenuations caused by human body are not considered. Previous studies have proposed WiFi fingerprinting positioning based on user orientation using compasses built in smartphones. However, compasses always cannot provide required accuracy of user orientation estimation due to the severe indoor magnetic perturbations. More importantly, we discover that not only user orientations but also smartphone carrying positions may affect signal attenuations caused by human body greatly. Therefore, we propose a novel WiFi fingerprinting positioning approach considering both user orientations and smartphone carrying positions. For user orientation estimation, we deploy Rotation Matrix and Principal Component Analysis (RMPCA) approach. For carrying position recognition, we propose a robust Random Forest classifier based on the developed orientation invariant features. Experimental results show that the proposed WiFi positioning approach may improve positioning accuracy significantly.
\end{abstract}

\section{Introduction}

Location awareness [1-5] based on pervasively available devices such as smartphones has received increasing attentions for Internet of Things (IoT) applications. The Global Navigation Satellite Systems (GNSS) [6] have dominated the outdoor positioning solutions. For indoor environments, since the GNSS signal cannot penetrate man-made infrastructures, many indoor positioning technologies, such as ultra-wideband [7] and radio frequency identification [8], have been developed. Most existing indoor positioning solutions with high positioning accuracy require extra hardware and high cost. Because of the large-scale deployment of access points (APs) and pervasively available smartphones with wireless network card installed, WiFi positioning using received signal strength (RSS) has been considered as one of the most popular indoor positioning solutions.

WiFi positioning systems can be divided into model based approach and fingerprinting based approach. The model based approach $[9,10]$ constructs the function relating RSS with the physical distance between the mobile devices and the AP. If more than three physical distances are obtained, user location estimation can be obtained by deploying a trilateration method. In contrast, the fingerprinting based approach [11-13] includes two phases: offline phase and online phase. During offline phase, RSS values from available APs are collected at predefined calibration points and stored as fingerprints to generate the so-called radio map. During online phase, the new measured RSS vectors are matched with the prestored fingerprints in the radio map. The user location is ultimately estimated by weighting locations of the matched calibration points $[14,15]$ or deploying some machine learning based methods $[16,17]$. Due to the complex radio propagation environment, it is difficult for model based approach to construct an accurate function to estimate the distance information. As a result, the accuracy performance cannot be guaranteed by the model based approach. Therefore, we deploy WiFi fingerprinting approach in our paper.

For WiFi fingerprinting approach, a key challenge is the RSS radio mismatch [18-20] phenomenon. This phenomenon occurs when radio environments between RSS radio map construction during offline phase and online positioning phase are obviously different and may deteriorate accuracy performance of WiFi fingerprinting positioning greatly. One main reason of radio mismatch is caused by the 
neglected user orientations [21]. Users tend to carry smartphones near to their body, which consists of more than $50 \%$ water. The human body might block WiFi signal transmission of $2.4 \mathrm{GHz}$, especially when the body is between an AP and a smartphone [22]. Previous works [23-25] have carried experiments to confirm the substantial RSS variations caused by user orientations. RSS may vary up to $15 \mathrm{dBm}$ [21] at a fixed location when the user facing an AP turns around. As a result, for a fingerprinting based approach, the mismatch of user orientations between offline training phase and online positioning phase may degrade the positioning accuracy greatly.

In order to solve the user orientation mismatch problem, in [26] a user orientation based fingerprinting positioning approach has been proposed. A digital compass built in a smartphone is utilized to detect the user orientation. During offline phase, the user orientation information is also added to enhance the radio map. During online phase, based on the estimated user orientation information, new measured RSS vector samples are only compared with the selected fingerprints sharing a similar user orientation. Similar approaches have been proposed in $[27,28]$, in which the radio map is extended with user orientation information. However, two problems still need to be solved to enhance the reliability and applicability of fingerprinting based positioning. Firstly, for indoor environments, Earth's magnetic field exploited by digital compass is always noisy and severely perturbed by manmade infrastructures, rendering the user orientation estimations using compasses unavailable. Secondly, we discover that not only user orientations but also smartphone carrying positions may affect RSS greatly due to the different human body attenuation schemes. The impact of different carrying positions on RSS patterns is not considered by previous works, which always assume that the smartphones are held in hand in front of users. However, for daily uses of a smartphone, other carrying positions $[29,30]$ such as in the trouser pocket or near to ear during phone calls are also widely deployed.

This paper proposes a novel WiFi fingerprinting positioning approach based on user orientation estimation and smartphone carrying position recognition. To the best of our knowledge, our research is the first to consider impacts of user orientation and smartphone carrying position information on RSS patterns simultaneously. Both the user orientation and the smartphone carrying position information are added into the radio map. For user orientation estimation, rather than deploy noisy digital compasses, we deploy our previous proposed Rotation Matrix and Principal Component Analysis (RMPCA) approach [31]. The basic principle is to exploit the principal component of accelerations in the horizontal plane, which is assumed to be parallel with user walking direction. For smartphone carrying position recognition, we investigate the four most classical positions [32], including being held in hand in front of the user (hand-held), put in the trouser pocket (inpocket), near to the right ear during phone calls (phone-call), and in the swinging-hand (swinging-hand). We deploy the Random Forest classifier [33] to recognize the four carrying positions. In order to adapt the changing device orientations, we exploit several orientation invariant features as input features of the classifier.
Experiments in a realistic indoor environment show that the proposed WiFi fingerprinting positioning approach may enhance reliability and applicability significantly. In summary, our work makes the main contributions as follows:

(i) We propose a novel WiFi fingerprinting approach considering both user orientations and smartphone carrying positions.

(ii) We discover that not only user orientations but also smartphone carrying positions may affect RSS patterns greatly.

(iii) We propose a robust carrying position recognition classifier by exploiting several orientation invariant features.

(iv) We report the evaluation of the proposed fingerprinting approach based on extensive experimental samples and compare it with existing approaches.

The remainder of this paper is structured as follows. We present an overview of the proposed WiFi fingerprinting positioning in the following section. In Section 3, we propose a robust carrying position classifier. Section 4 describes the user orientation estimation algorithm using RMPCA approach. Section 5 firstly introduces the impact of user orientations and carrying positions on RSS and then presents the proposed user orientation and carrying position based fingerprinting approach. The experimental results are reported and analyzed in detail in Section 6. In the last section, we give a conclusion and our future works.

\section{Overview of the Proposed WiFi Fingerprinting Positioning Approach}

Figure 1 presents an overview of the proposed WiFi fingerprinting positioning approach based on user orientation estimation and carrying position recognition. The approach is divided into offline phase and online phase. During offline phase, RSS data at predefined calibration points are collected at eight orientations equally distributed in a circle with four investigated different carrying positions, respectively. As a result, an enhanced radio map, whose fingerprints are labeled with both user orientations and carrying positions, is constructed.

During online phase, we deploy a robust Random Forest based classifier to recognize the smartphone carrying positions. The classifier exploits orientation invariant features generated by acceleration signals as input features. Furthermore, we develop RMPCA based user orientation estimation upon a continuous device attitude tracking. Using the estimated user orientations and recognized carrying positions, we select the fingerprints with similar orientation and the same carrying position, instead of all the fingerprints in the enhanced radio map. Finally, a pattern matching algorithm based on maximum likelihood [22] is used for ultimate location estimation.

\section{Robust Smartphone Carrying Position Recognition}

For daily uses of smartphones, it is more applicable to assume an unfixed carrying position. For example, it is more common for male users to put the smartphones in their trouser pockets 


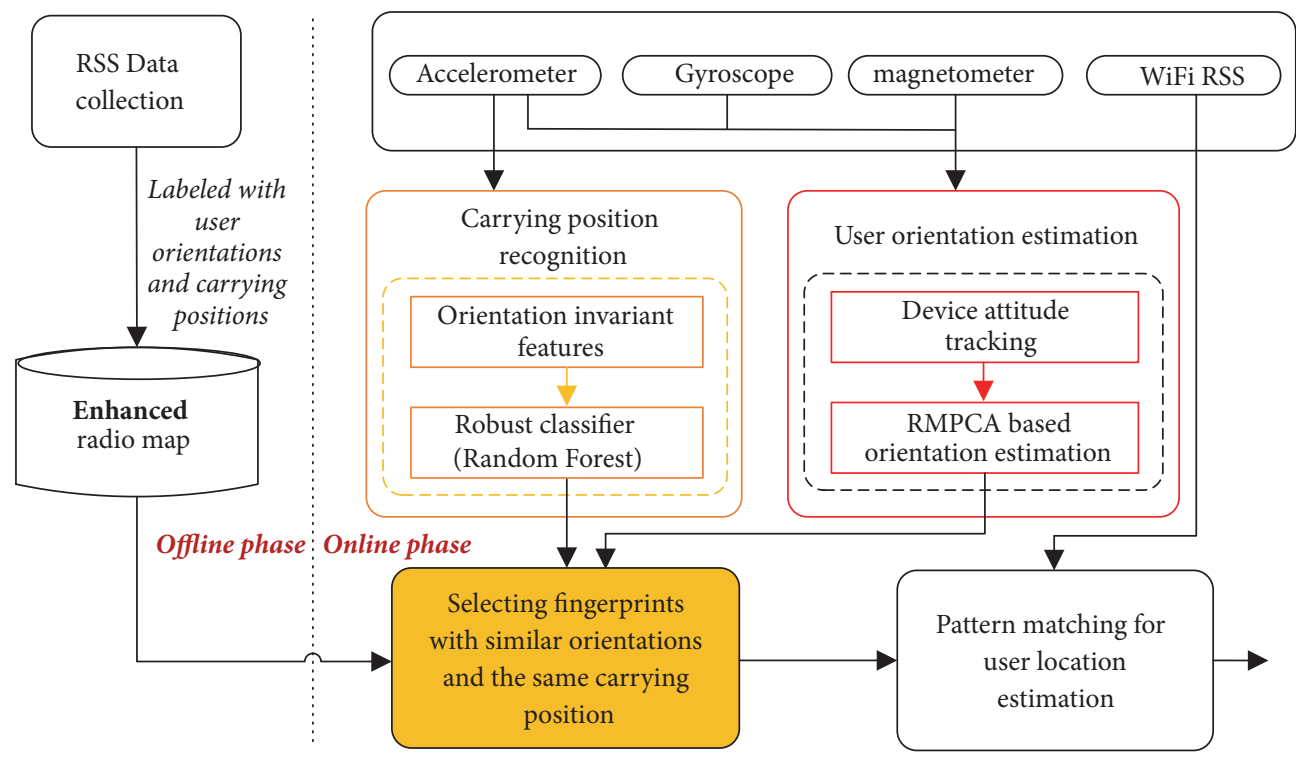

FIGURE 1: Overview of the proposed WiFi fingerprinting positioning approach.

[29]. Different RSS values will be collected with different carrying positions, even in a fixed location with the same user orientation, as will be seen later in Section 5.1. Therefore, we develop a smartphone carrying position recognition method to detect the device carrying position before pattern matching of fingerprinting based approach. For simplicity, we only investigate the four most widely used carrying positions: hand-held, inpocket, swinging-hand, and phone call.

As previous works [34, 35] suggested, acceleration signals with different device carrying positions show different patterns. Previous classifiers are always founded on relatively fixed and controlled device orientations and deploy normal time domain features [34] for carrying position classifier. The statistics of raw acceleration signal samples over a sliding window, including maximum, minimum, mean, medium, and variance, are computed and deployed as input features to train the carrying position classifier. However, the device orientations may vary for different users due to different user habits. For the same user, the device orientation may also change with the user walking and the other body locomotion. If the device orientations are not fixed, the threedimensional acceleration signals may vary a lot with unconstrained device orientations at a fixed carrying position. As a result, classification accuracy of previous carrying position classifiers may deteriorate significantly with unconstrained device orientations.

In order to recognize carrying positions with unconstrained device orientations, we propose a robust classifier upon orientation invariant features. Instead of deploying raw three-dimensional accelerations, we exploit three variables, including total magnitude of acceleration $M A c c$, magnitude of the horizontal acceleration $H A c c$, and acceleration in the gravity direction $G A c c$,

$$
M A c c=|\mathbf{A c c}|_{2}=\sqrt{A c c_{x}^{2}+A c c_{y}^{2}+A c c_{z}^{2}}
$$

$$
\begin{aligned}
& H A c c=\left|A c c-\left(A c c \cdot \mathbf{g}_{\text {normal }}\right) \mathbf{g}_{\text {normal }}\right|_{2} \\
& \text { GAcc }=\left|\left(A c c \cdot \mathbf{g}_{\text {normal }}\right) \mathbf{g}_{\text {normal }}\right|_{2}
\end{aligned}
$$

where $\mathbf{A c c}=\left[\begin{array}{llll}A c c_{x} & A c c_{y} & A c c_{z}\end{array}\right]^{T}$ is the raw acceleration signal, $|\cdot|_{2}$ is the 2-norm operator, and $\mathbf{g}_{\text {normal }}$ is related gravity vector with normalized value. The gravity vector can be obtained by transforming standard gravity vector using tracked quaternion vector as in (4). These variables show distinct patterns at different carrying positions. Compared with raw acceleration signals, these variables remain relatively stable when the carrying positions are the same, regardless of changing device orientations.

The classifier design includes three stages: data preprocessing, feature extraction, classifier training, and testing. Firstly, we collect raw acceleration signal samples from an accelerometer continuously and split them into segments by a sliding window [34]. Size of the window is two seconds and with one second overlap between neighboring windows. Then, we compute three-orientation invariant variable samples over each window. After data preprocessing, we exploit the statistics of the three variables as input features of carrying positioning classifier, including maximum, minimum, mean, medium, and variance. The input features are all useful to distinguish different carrying positions. For example, because of the higher locomotion intensity of leg than the upper user body, maximum values of HAcc for inpocket are always significantly larger than those of phone-call positions. Variance values of $M A c c$ of the phone-call positions are always significantly smaller than those of inpocket positions. More importantly, these input features are insensitive to device orientations and almost independent with device orientations. 
After feature extraction, we deploy Random Forest as the classifier, which performs well in many multiclass classification problems. We collect acceleration signals over four thousand sliding windows with four investigated carrying positions. For each position, we sample acceleration signals over all possible device orientations uniformly. We use the widely used 10-fold cross-validation method to train and test the classifier. The extracted features are partitioned into ten parts randomly. Nine of the ten parts are used for training and the rest part for testing. We repeat the whole process for one hundred times by randomly selecting one part for testing. Experimental results show that the robust Radom Forest classifier may improve classification accuracy than compared previous classifiers, as will be seen in Section 6.2.

\section{User Orientation Estimation}

In order to obtain and represent the absolute user orientation, we firstly define global coordinate system (GCS). GCS consists of three axes $X_{G}, Y_{G}$, and $Z_{G}$, which point to east, north, and the sky, respectively. To describe the raw measured signals from inertial sensors, we also define device coordinate system (DCS), which consists of three axes $X_{D C S}, Y_{D C S}$, and $Z_{D C S}$. The $X_{D C S}$ and $Y_{D C S}$ axes point right and forward in the front face of smartphone screen, respectively, while $Z_{D C S}$ axis is a standard cross-product of the former two axes. We assume that the initial user orientation is known by other techniques such as landmarks [36]. The user orientation is just the user walking direction, whose value at GCS is defined as the rotation angle from the positive $Y_{G}$ to user walking direction counterclockwise.

4.1. Device Attitude Tracking. For RMPCA based user orientation approach, it is important to obtain the device attitude continuously. We deploy Extended Kalman Filter (EKF) to model the device attitude tracking. Before designing EKF, we describe device attitude by quaternion vector as given,

$$
\mathbf{h}^{D C S}(t)=\left(\mathbf{C}_{G C S}^{D C S}(\mathbf{q}(t))\right)^{T} \mathbf{h}^{G C S}(t)
$$

where $\mathbf{C}_{G C S}^{D C S}(\mathbf{q}(t))$ is the rotation matrix from DCS to GCS, $\mathbf{h}^{G C S}(t)$ and $\mathbf{h}^{D C S}(t)$ are the vectors represented at GCS and DCS, and argument $t$ represents the time, which will be omitted in the following equations, rotation matrix can be given as

$$
\begin{aligned}
& \mathbf{C}_{G C S}^{D C S}(\mathbf{q}) \\
& =\left[\begin{array}{ccc}
q_{0}^{2}+q_{1}^{2}-q_{2}^{2}-q_{3}^{2} & 2\left(q_{1} q_{2}-q_{0} q_{3}\right) & 2\left(q_{1} q_{3}+q_{0} q_{2}\right) \\
2\left(q_{1} q_{2}+q_{0} q_{3}\right) & q_{0}^{2}-q_{1}^{2}+q_{2}^{2}-q_{3}^{2} & 2\left(q_{2} q_{3}-q_{0} q_{1}\right) \\
2\left(q_{1} q_{3}-q_{0} q_{2}\right) & 2\left(q_{0} q_{1}+q_{2} q_{3}\right) & q_{0}^{2}-q_{1}^{2}-q_{2}^{2}+q_{3}^{2}
\end{array}\right]
\end{aligned}
$$

where $\mathbf{q}=\left[\begin{array}{llll}q_{0} & q_{1} & q_{2} & q_{3}\end{array}\right]^{T}$ is the quaternion vector with normalized magnitude.
Secondly, following the kinematic law of rigid body [37], the discrete time evolution model of rotation quaternion vector is given as follows:

$$
\begin{aligned}
& \mathbf{q}_{k+1}=\exp \left(0.5 \times \Omega\left(\mathbf{w}_{k} T_{\mathrm{s}}\right)\right) \mathbf{q}_{k} \\
& \quad=\left(\mathbf{I} \cos \left(0.5 \times \Delta \theta_{k}\right)+\frac{\Omega\left(\mathbf{w}_{k} T_{s}\right) \sin \left(0.5 \times \Delta \theta_{k}\right)}{\Delta \theta_{k}}\right) \mathbf{q}_{k}
\end{aligned}
$$

where $T_{s}$ is the time interval, $k$ and $k+1$ represent time instants $k T_{s}$ and $(k+1) T_{s}, \mathbf{q}_{k}$ and $\mathbf{q}_{k+1}$ are the related time evolution quaternion vectors, $\mathbf{w}_{k}=\left[\begin{array}{lll}w_{k}^{x} & w_{k}^{y} & w_{k}^{z}\end{array}\right]^{T}$ is threedimensional angular velocity at DCS, I is an $3 \times 3$ identity matrix, $\Delta \theta_{k}=T_{s} \sqrt{\left(w_{k}^{x}\right)^{2}+\left(w_{k}^{y}\right)^{2}+\left(w_{k}^{z}\right)^{2}}$, and $\Omega\left(\mathbf{w}_{k} T_{s}\right)$ is given by

$$
\Omega\left(\mathbf{w}_{k} T_{s}\right)=T_{s}\left[\begin{array}{cccc}
0 & -w_{k}^{x} & -w_{k}^{y} & -w_{k}^{z} \\
w_{k}^{x} & 0 & w_{k}^{z} & -w_{k}^{y} \\
w_{k}^{y} & -w_{k}^{z} & 0 & w_{k}^{x} \\
w_{k}^{z} & w_{k}^{y} & -w_{k}^{x} & 0
\end{array}\right]
$$

The quaternion $\mathbf{q}_{k+1}$ is determined once the initial quaternion vector $\mathbf{q}_{0}$ is known. Therefore, state model of EKF is given by

$$
\mathbf{q}_{k+1}=F_{k} \mathbf{q}_{k}+\mathbf{w}_{k}^{q}
$$

where the state transition matrix $F_{k}=\exp \left(0.5 * \Omega\left(\mathbf{w}_{k} T_{\mathrm{s}}\right)\right)$ and

$$
\mathbf{w}_{k}^{q}=\Xi_{k} \mathbf{w}_{k}^{\text {gyro }}=-\frac{T_{s}}{2}\left[\begin{array}{c}
{\left[\mathbf{e}_{k} \times\right]+q_{0}^{k} \mathbf{I}} \\
-\mathbf{e}_{k}^{T}
\end{array}\right] \mathbf{w}_{k}^{\text {gyro }}
$$

$\mathbf{q}_{k}=\left[\begin{array}{llll}q_{0}^{k} & q_{1}^{k} & q_{2}^{k} & q_{3}^{k}\end{array}\right]^{T}$ is the rotation quaternion vector at time instants $k T_{s}$ with vector part $\mathbf{e}_{k}=\left[\begin{array}{lll}q_{1}^{k} & q_{2}^{k} & q_{3}^{k}\end{array}\right]^{T}, \mathbf{w}_{k}^{\text {gyro }}$ is the Gaussian measurement noise variable of angular velocity, and $\left[\mathbf{e}_{k} \times\right]$ is a standard vector cross-product operator.

The measurement model of EKF is given by

$$
\begin{aligned}
\mathbf{z}_{k+1}= & {\left[\begin{array}{c}
\mathbf{a}_{k+1} \\
\mathbf{m}_{k+1}
\end{array}\right]=\phi\left(\mathbf{q}_{k+1}\right)+\mathbf{v}_{k+1} } \\
= & {\left[\begin{array}{cc}
\left(\mathbf{C}_{G C S}^{D C S}\left(\mathbf{q}_{k+1}\right)\right)^{T} & \mathbf{0} \\
\mathbf{0} & \left(\mathbf{C}_{G C S}^{D C S}\left(\mathbf{q}_{k+1}\right)\right)^{T}
\end{array}\right]\left[\begin{array}{l}
\mathbf{g}_{0} \\
\mathbf{h}_{0}
\end{array}\right] } \\
& +\left[\begin{array}{c}
\mathbf{v}_{k+1}^{a} \\
\mathbf{v}_{k+1}^{m}
\end{array}\right]
\end{aligned}
$$

where $\mathbf{a}_{k+1}$ and $\mathbf{m}_{k+1}$ are the raw measured acceleration and magnetic vectors at DCS, respectively; $\mathbf{v}_{k+1}^{a}$ and $\mathbf{v}_{k+1}^{m}$ are the related measurement noise variables; $\mathbf{g}_{0}$ and $\mathbf{h}_{0}$ are the local gravity and magnetic vectors at GCS, respectively. The measurement noise covariance matrix $\mathbf{R}_{k+1}$ is given by

$$
\mathbf{R}_{k+1}=\left[\begin{array}{cc}
\mathbf{R}_{k+1}^{a} & \mathbf{0} \\
\mathbf{0} & \mathbf{R}_{k+1}^{m}
\end{array}\right]=\left[\begin{array}{cc}
R \sigma_{a}^{2} \mathbf{I}_{3} & \mathbf{0} \\
\mathbf{0} & R \sigma_{m}^{2} \mathbf{I}_{3}
\end{array}\right]
$$


where ${ }^{R} \sigma_{a}^{2}$ and ${ }^{R} \sigma_{m}^{2}$ are set adaptively as follows:

$$
\begin{aligned}
& { }_{\sigma_{a}^{2}}= \begin{cases}\sigma_{a}^{2}, & \left|\left\|\mathbf{a}_{k+1}\right\|_{2}-\left\|\mathbf{g}_{0}\right\|_{2}\right|<\varepsilon_{a 1} \cap \operatorname{var}\left(\left\|\mathbf{a}_{k+1-N a / 2}\right\|_{2}:\left\|\mathbf{a}_{k+1+N a / 2}\right\|_{2}\right)<\varepsilon_{a 2} \\
\infty, & \text { otherwise }\end{cases} \\
& { }^{R} \sigma_{m}^{2}= \begin{cases}\sigma_{m}^{2}, & \left\|\mathbf{m}_{k+1}\right\|_{2}-\left\|\mathbf{h}_{0}\right\|_{2} \mid<\varepsilon_{m 1} \cap \operatorname{var}\left(\left\|\mathbf{m}_{k+1-N m / 2}\right\|_{2}:\left\|\mathbf{m}_{k+1+N m / 2}\right\|_{2}\right)<\varepsilon_{m 2} \\
\infty, & \text { otherwise }\end{cases}
\end{aligned}
$$

where $\varepsilon_{a 1}$ is the allowed maximum difference between magnitude of new measured acceleration signal and that of the local gravity vector, $\operatorname{var}(\cdot)$ calculates the variance of signals within a centered window, $\varepsilon_{a 2}$ is the allowed maximum variance of $N_{a}$ acceleration signals, $\varepsilon_{m 1}$ is the allowed maximum difference between magnitude of new measured magnetic signal and that of the local magnetic signal, and $\varepsilon_{m 2}$ is the allowed maximum variance of $N_{m}$ magnetic signals.

Based on the state model in (8) and the measurement model in (10), the state vector $\mathbf{q}_{k+1}$ can be iteratively updated by EKF model.

4.2. RMPCA Based User Orientation Estimation. Many works [38] assume that the user orientation is the same as the device forward direction or the angle difference between them is fixed. Therefore, they can directly estimate the user orientation using the device attitude tracking model described in Section 4.1. However, the angle difference between user orientation and device forward direction always varies rapidly with highly dynamic body locomotion, rendering attitude estimation inapplicable for user orientation estimation. Therefore, we deploy RMPCA approach [31] for user orientation estimation. During pedestrian walking period, we assume that the user orientation is just the walking direction. The basic principle of RMPCA is that walking direction is parallel to the principal component of the acceleration signals in the horizontal plane, which dominates most of the horizontal acceleration signal energy.

In detail, RMPCA firstly transforms all raw measured RSS signals during a walking step at DCS into GCS, as follows:

$$
\mathbf{a}_{G C S}^{i}=\mathbf{C}_{G C S}^{D C S}(\mathbf{q}) \mathbf{a}_{D C S}^{i}, \quad i=1, \ldots, N_{\text {step }}
$$

where $\mathbf{a}_{G C S}^{i}=\left[\begin{array}{lll}\mathbf{a}_{G C S}^{i}(x) & \mathbf{a}_{G C S}^{i}(y) & \mathbf{a}_{G C S}^{i}(z)\end{array}\right]^{T}$ is the $i-$ th acceleration signal represented at GCS, $N_{\text {step }}$ is the number of acceleration signals within the walking step, and $\mathbf{q}$ is the tracked quaternion vector. Each walking step is detected by a widely used peak detection algorithm [39], which relates the heel strike of each step to an acceleration peak value. The acceleration signals in the horizontal plane are in fact the former two acceleration components of $\mathbf{a}_{G C S}^{i}$. Then, we perform PCA over horizontal acceleration signals within a walking step to extract the walking direction $\mathbf{W D}_{\text {global }}=$
$\left[W D_{\text {global }}^{x} W D_{\text {global }}^{y}\right]^{T}$. The user orientation $O_{\text {user }}$ can be estimated as follows:

$$
O_{\text {user }}=\arctan \left(\frac{W D_{\text {global }}^{y}}{W D_{\text {global }}^{x}}\right)-\frac{\pi}{2}
$$

\section{Proposed WiFi Fingerprinting Positioning Approach}

This section firstly describes the impact of user orientations and carrying positions on RSS, which may directly indicate the motivation of the proposed approach. Then, we describe the proposed fingerprinting approach based on an enhanced radio map considering both user orientations and carrying positions. The user location is ultimately estimated by a maximum likelihood based pattern matching algorithm.

5.1. Impact of User Orientations and Carrying Positions on RSS. We have carried four experiments to evaluate the impact of user orientations and carrying positions on RSS: line-of-sight (LOS) environment near to AP, LOS environment far away from AP, none-line-of-sight (NLOS) environment near to AP, and NLOS environment far away from AP. We program a smartphone to collect RSS readings every 500 milliseconds from an AP with a total 200 samples for each fixed user orientation and carrying position. The experimental user rotates its orientation by an interval of 45 degrees for a total number of eight orientations. For near and far environments, the distances between AP and user are four and twelve meters, respectively. For user orientation of 90 degrees and 180 degrees, the user is faced with and points away from the AP, respectively.

Figure 2 shows the mean RSS of different user orientations with four carrying positions under the LOS environment near to AP. For hand-held position, RSS values of the user just facing to AP or with the adjacent orientations are greatly larger than those of back toward AP. In particular, compared with user orientation of 180 degrees (back toward AP), the mean RSS values with user orientation of 90 degrees (facing to AP) increase about $20 \mathrm{~dB}$. This is because a user holding the smartphone in hand may block the LOS WiFi signal totally when he stands between the AP and the smartphone. Similarly, for hand-held position under LOS environment far away from AP, RSS values of the user just faced with AP or with the adjacent orientations are also significantly larger 


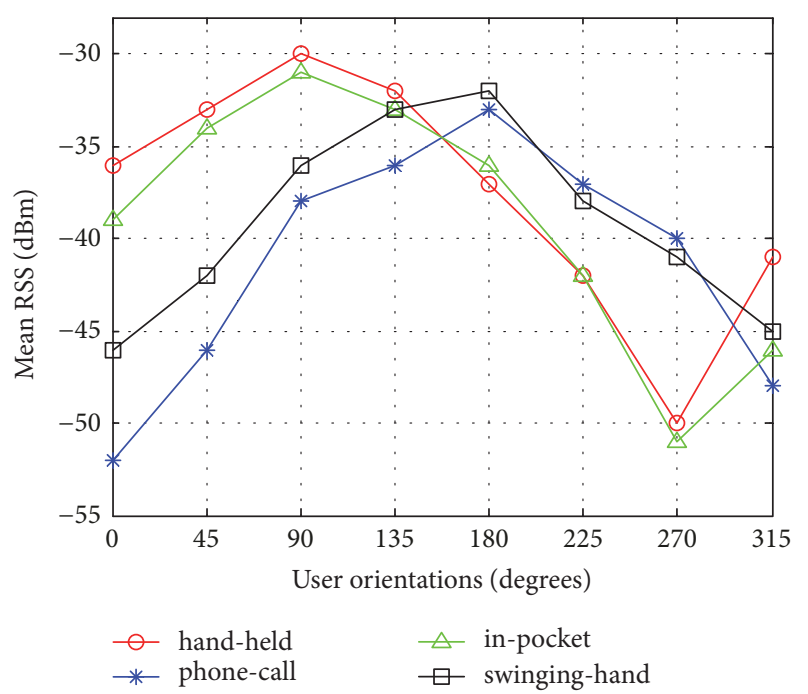

FIGURE 2: Impact of user orientations and carrying positions on mean RSS under the LOS environment near to AP.

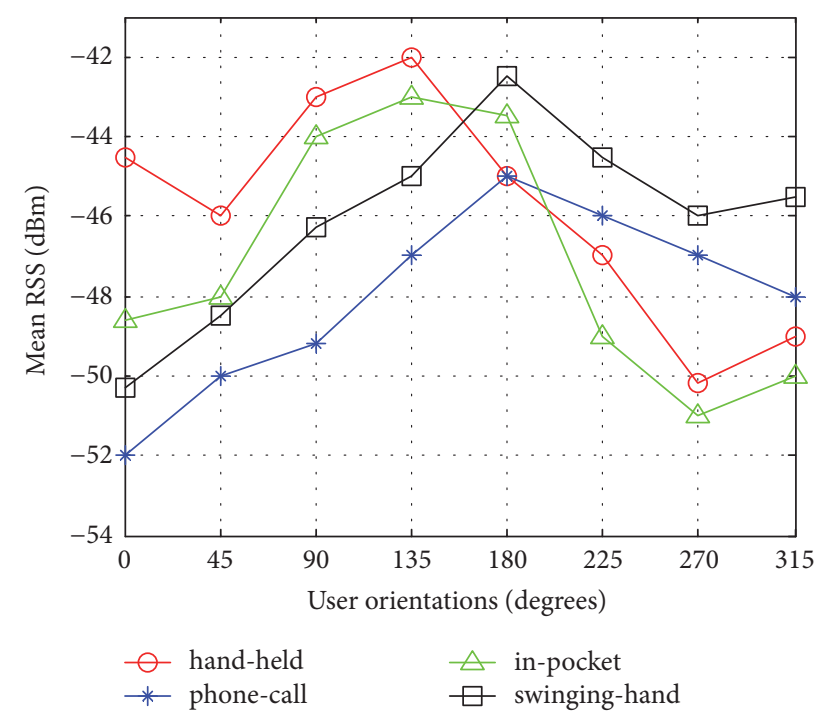

FIGURE 3: Impact of user orientations and carrying positions on mean RSS under the NLOS environment near to AP.

than those of back toward AP, while the increments are a little smaller than those of the near to AP environment.

For the other three carrying positions, there are also significant RSS variations caused by varied user orientations. However, the maximum and minimum RSS values may occur in a very different user orientation. For the phonecall position, the minimum RSS value occurs in the user orientation of 0 degrees instead of 270 degrees for the handheld position, since the side of user body rather than the whole user body blocks the LOS WiFi signal. As a result, RSS patterns of the same user orientation and different carrying positions are also distinct due to the different signal blocking scheme of user body. It is necessary to add both user orientations and carrying positions to enhance the radio map.

Figure 3 shows the mean RSS of different user orientations with four carrying positions under the NLOS environments near to AP. We can see that, for hand-held position, RSS values of the user just facing to AP increase about $8 \mathrm{~dB}$ than those of back toward AP. Since there is no direct path between AP and the smartphone, the blocking effect of user body under NLOS environment is significantly smaller than that of LOS environment. Furthermore, for NLOS environment far away from AP, the signal blocking effect of user body can be neglected, due to the more complex multipath and NLOS propagation environments. However, for different user orientations and carrying positions, RSS patterns are also different due to different radio propagation environments, though there are no obvious effects of signal absorptions caused by user body. Therefore, in order to avoid introducing the radio mismatch during pattern matching process, both user orientations and carrying positions should be considered in the WiFi fingerprinting approach.

5.2. Enhanced Radio Map Based Location Estimation Using Maximum Likelihood. The proposed WiFi fingerprinting positioning approach consists of two stages: offline enhanced radio map construction and online location estimation. During the offline stage, RSS readings from multiple available APs are collected to build the radio map. The raw RSS value is denoted as $\psi_{i, j}^{o, c}[\tau]$, which indicates RSS from the $i-$ th AP at the $j-$ th calibration point, with user orientation $o$ and carrying position $c$. For each calibration point, we collect RSS data at eight orientations $O=\left\{0^{\circ}, 45^{\circ}, \ldots, 315^{\circ}\right\}$ with four different carrying positions $C=$ hand-held, in-pocket, swinging-hand, phone-call $\}$. Then, the enhanced radio map can be represented by $\psi$ as

$$
\psi^{o, c}[\tau]=\left(\begin{array}{cccc}
\psi_{1,1}^{o, c}[\tau] & \psi_{1,2}^{o, c}[\tau] & \cdots & \psi_{1, N}^{o, c}[\tau] \\
\psi_{2,1}^{o, c}[\tau] & \psi_{2,2}^{o, c}[\tau] & \cdots & \psi_{2, N}^{o, c}[\tau] \\
\vdots & \vdots & \ddots & \vdots \\
\psi_{M, 1}^{o, c}[\tau] & \psi_{M, 2}^{o, c}[\tau] & \cdots & \psi_{M, N}^{o, c}[\tau]
\end{array}\right)
$$

where $M$ is the number of APs, $N$ is the number of calibration points, $\tau=1, \ldots, Q$ is time index of temporal RSS samples, and $Q$ is the number of RSS samples. The row vector of $\psi$ represents the RSS data of one AP over $N$ calibration points with $Q$ temporal length. The column vector of $\psi$ represents the $M$-dimensional RSS samples at the $j-$ th calibration point.

During online stage, a maximum likelihood based pattern matching algorithm is used for location estimation. Maximum likelihood algorithm estimates the likelihood value of new measured RSS observations for all candidate calibration points and weights the points having maximum likelihood as the positioning result. The new measured RSS vectors can be represented as

$$
\psi_{\text {new }}^{\tilde{o}, \tilde{c}}=\left[\begin{array}{llll}
\psi_{1, \text { new }}^{\tilde{o}, \tilde{c}} & \psi_{2, \text { new }}^{\tilde{o}, \tilde{c}} & \cdots & \psi_{M, \text { new }}^{\tilde{o}, \tilde{c}}
\end{array}\right]^{T}
$$

where $\widetilde{o}$ is the estimated user orientation as in (15) and $\widetilde{c}$ is the recognized carrying position of the four investigated positions. Based on the estimated user orientation 


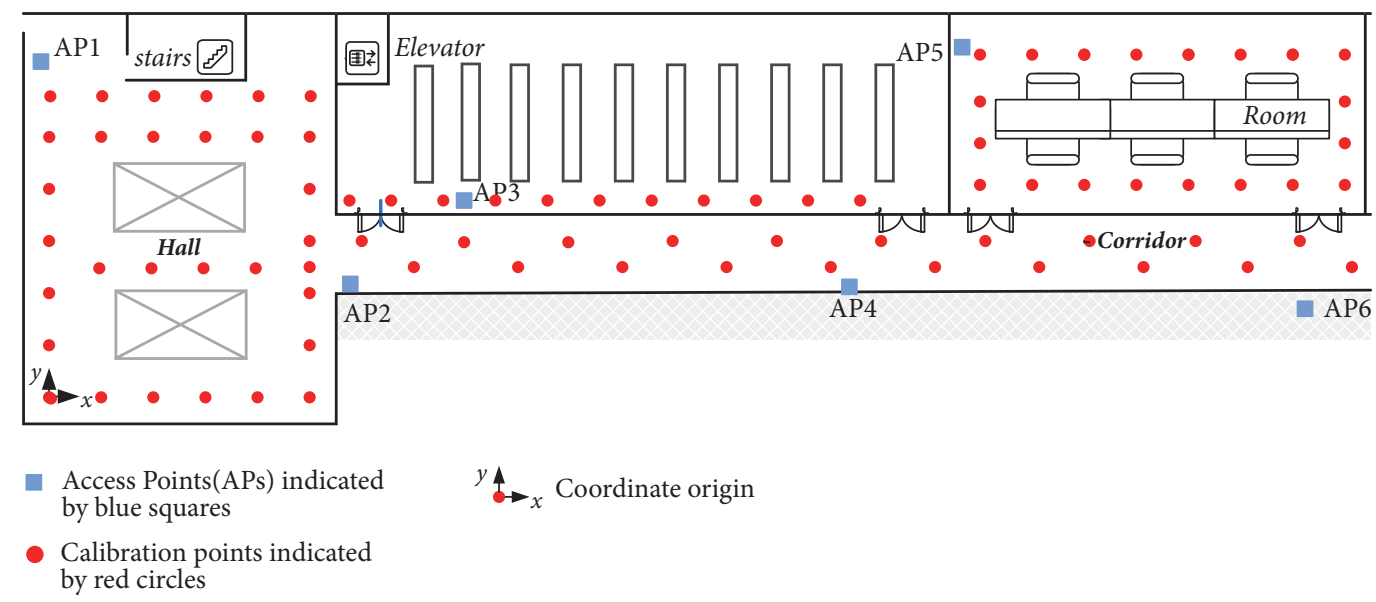

FIGURE 4: Realistic indoor experimental environments for WiFi positioning.

and recognized carrying position, we select the fingerprints with similar orientations and the same positions for pattern matching. Particularly, we obtain the two orientations $o 1, o 2$ in the set $O$, which have the smallest absolute differences with the estimated user orientation $\widetilde{o}$. The selected radio map can be generated by selecting fingerprints $\psi^{o 1, \widetilde{c}}[\tau]$ and $\psi^{o 2, \widetilde{c}}[\tau]$. For each calibration point, RSS values collected at two orientations $o 1, o 2$ are merged into one group.

Maximum likelihood algorithm treats the statistical distribution of RSS from one AP at each calibration point as Gaussian distribution. For new RSS vectors, the likelihood at calibration point $L_{i}$ is calculated by the product of likelihood of all available APs:

$$
f\left(\psi_{\text {new }}^{\tilde{\sigma}, \tilde{c}} \mid L_{i}\right)=\prod_{j=1}^{M} f\left(\psi_{j, \text { new }}^{\tilde{\sigma}, \tilde{c}} \mid L_{i}\right)
$$

where $f\left(\psi_{j, \text { new }}^{\widetilde{o}, \widetilde{c}} \mid L_{i}\right)$ is the likelihood of calibration point $L_{i}$ for the $j$-th AP. The Gaussian function is deployed as likelihood function for RSS statistical distribution:

$$
f\left(\psi_{j, \text { new }}^{\tilde{o}, \tilde{c}} \mid L_{i}\right)=\frac{1}{\sqrt{2 \pi} \sigma} \exp \left(-\frac{\left(\psi_{j, \text { new }}^{\tilde{\rho}, \tilde{c}}-\mu_{i}^{j}\right)^{2}}{2 \sigma^{2}}\right)
$$

where $\mu_{i}^{j}$ is the mean RSS value for the $j-$ th AP at calibration point $L_{i}$ with carrying position $\widetilde{c}$ and the user orientations $o 1, o 2$ and $\sigma$ is the parameter relating with standard variance. The user location is ultimately estimated by averaging the $K$ calibration points,

$$
L_{\text {user }}=\frac{1}{K} \sum_{i=1}^{K} \widetilde{L}_{i}
$$

where $\widetilde{L}_{i}$ are the locations of $K$ calibration points with the maximum likelihood value.

\section{Evaluation}

6.1. Experimental Setup. We performed experiments in a typical realistic indoor environment, whose size is $27.4 \mathrm{~m}$ $\times 11.2 \mathrm{~m}$, as shown in Figure 4. We deploy a Samsung Galaxy S4 smartphone as experimental device with wireless network card, gyroscope, accelerometer, and magnetometer installed. For offline radio map construction, we collect RSS vector samples from all available APs at 82 calibration points with eight user orientations and four investigated carrying positions. The eight user orientations are set along the four coordinate axes directions and four middle directions of them. For each calibration point, 3200 samples are collected with 100 samples per user orientation and carrying position. The calibration points are approximately uniformly distributed in the target environment with a distance of about one meter along the $x$-axis direction. A total number of eight APs are available, with six APs seen in the target environment and the rest ones in the adjacent area. For testing points, the participant walked along a path with one of the four investigated carrying positions reaching almost all coverage area of the calibration points. Six different walking paths with relatively different user orientations were carried. Each walking path is about one hundred meters long reaching most of the target environment and repeated ten times for each carrying position. The user true locations are obtained by placing multiple tags on the floor and deploying a video to record the walking path. For each experimental run, we assumed that the initial user orientation was known and the inertial sensors were already well calibrated [31].

6.2. Carrying Position Recognition Results. We collect one thousand sliding window samples for each carrying position and a total number of four thousand samples are used to train and test the classifiers. In order to make the classifier more robust to the changing device orientation, we collect acceleration signals under all possible device orientations as uniformly as possible. To avoid introducing noisy acceleration signals, we deploy a five-point average filter to smooth them.

Table 1 compares the classification accuracy performance of the proposed orientation invariant features and normal time domain features. The results show that the proposed robust carrying position classifier shows an average accuracy 
TABLE 1: Confusion table of Random Forest classifier with orientation invariant and normal features.

\begin{tabular}{lcccc}
\hline (Orientation Invariant/Normal Features) & Hand-Held & Phone-Call & Swinging-Hand & In-Pocket \\
\hline Hand-Held & $0.982 / 0.904$ & $0.018 / 0.063$ & $0 / 0.033$ & $0 / 0$ \\
Phone-Call & $0.01 / 0.064$ & $0.990 / 0.915$ & $0 / 0.004$ & $0 / 0.017$ \\
Swinging-Hand & $0 / 0.018$ & $0 / 0.001$ & $0.986 / 0.896$ & $0.014 / 0.085$ \\
In-Pocket & $0 / 0$ & $0 / 0$ & $0.028 / 0.081$ & $0.972 / 0.919$ \\
\hline
\end{tabular}

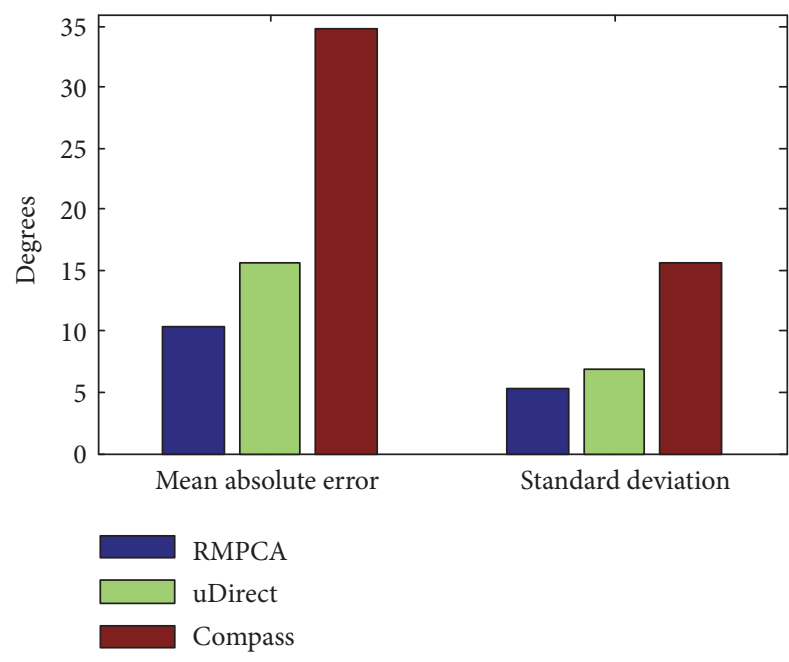

FIGURE 5: Mean absolute user orientation estimation error and the standard deviation comparisons between RMPCA, uDirect, and compass approaches.

reaching 98.5\%, which is high enough for recognizing the carrying position accurately. Therefore, the subsequent experiments assume that the used carrying position information is correctly recognized initially. Compared with the traditional normal time domain features, the proposed classifier achieves a significant accuracy improvement from $90.9 \%$ to $98.5 \%$. The improvement can be contributed to the higher discrimination and generalization ability of the extracted orientation invariant features. Rather than previous normal time domain features vary greatly with the changing device orientation, the extracted features keep relatively stable. Besides, the probabilities of confusing between swinginghand and inpocket pairs, hand-held, and phone-call pairs are higher than the other pairs, since the acceleration patterns within these pairs are more confused than the other pairs, due to their more similar patterns of body locomotion.

6.3. User Orientation Estimation Results. Figure 5 compares mean absolute user orientation estimation error and the standard deviation values between RMPCA, uDirect [40], and compass [26] approaches. The deployed RMPCA approach decreases the mean absolute estimation error by 33.8 percent (5.3 degrees) and 70.2 percent (24.4 degrees) more than those of the compared uDirect and compass approaches, respectively. For standard deviation, RMPCA approach decreases by 22.6 percent (1.6 degrees) and 66.1 percent (10.4 degrees) more than those of the compared uDirect and compass approaches, respectively. Clearly, the deployed RMPCA approach achieves the best user heading estimation accuracy.

For the uDirect approach, the user orientation during a walking period is extracted at the moment when acceleration signals along the waling direction dominate the accelerations in the horizontal plane. Unfortunately, such a moment is susceptible to noisy acceleration signals. Instead of exploiting some specific acceleration samples by uDirect approach, the RMPCA approach exploits all acceleration samples during a walking period by performing PCA over the horizontal acceleration signals. Therefore, the RMPCA approach is more robust and achieves smaller user orientation estimation error and standard deviation than the uDirect approach. For user orientation using a compass, since the indoor magnetic perturbations are severe and is difficult to be calibrated, the accuracy performance of user orientation is much worse than the two other approaches.

6.4. Location Estimation Results. This section compares the proposed WiFi positioning approaches considering user orientations and carrying positions; WiFi positioning approach based on user orientation estimation using a compass and traditional WiFi positioning approach do not consider these information. We perform traditional WiFi positioning approach by selecting fingerprints with random user orientations and carrying positions to construct the radio map. The pattern matching algorithm deploys the widely used maximum likelihood method. The positioning error is defined as the Euclidean distance between the estimated coordinates per step and the labeled coordinates per step along the true path. Positioning accuracy is mainly evaluated by the accumulative probability distribution of positioning errors.

As seen in Figure 6, accumulative error distribution within $1 \mathrm{~m}$ of the proposed approach is $35.1 \%$, while those of approach (compass) and traditional approach are $20.1 \%$ and $17.1 \%$, respectively. Accumulative error distribution within $3 \mathrm{~m}$ of the proposed approach is $88.9 \%$, while those of approach (compass) and traditional approach are $63.6 \%$ and $57.9 \%$, respectively. Table 2 also indicates error reduction of the proposed approach than the other two compared approaches. Compared with approach (compass) and traditional approach, the proposed approach decreases mean positioning errors by $41.7 \%(1.15 \mathrm{~m})$ and $46.7 \%(1.41 \mathrm{~m})$, respectively.

Compared with approach (compass) and traditional approach, the proposed approach obtains significant positioning accuracy improvement. This is because the proposed positioning approach may exploit the user orientation and carrying position information to enhance the radio map 
TABLE 2: Positioning error (meters) comparisons.

\begin{tabular}{lccc}
\hline Compared Approaches & Proposed approach & Approach (Compass) & Traditional Approach \\
\hline Mean error & 1.61 & 2.76 & 3.02 \\
75 percentile & 2.35 & 3.87 & 4.42 \\
90 percentile & 3.11 & 5.68 & 6.05 \\
Standard variance & 0.40 & 0.48 & 0.53 \\
\hline
\end{tabular}

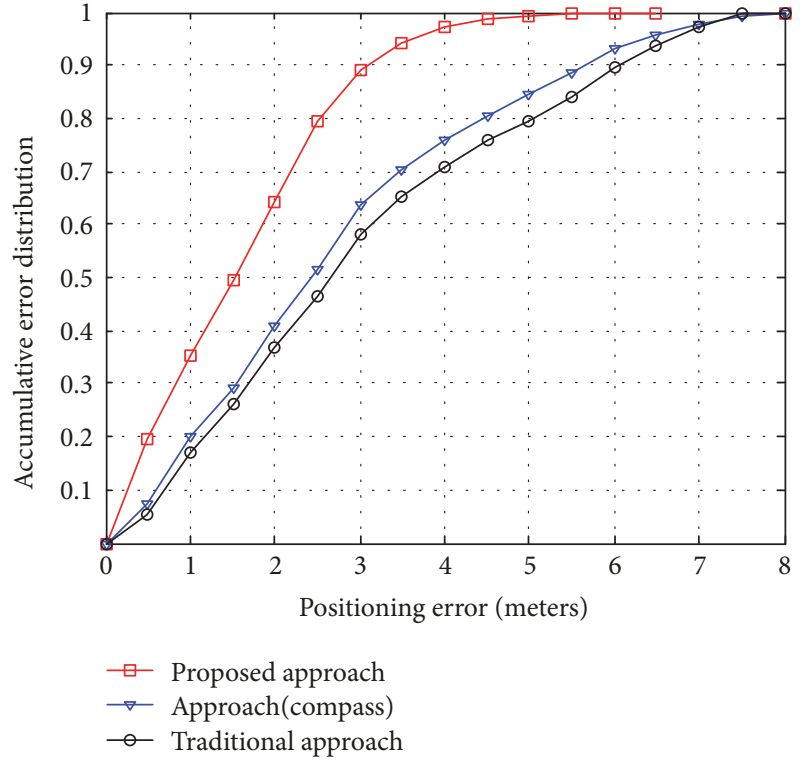

Figure 6: Accuracy comparisons between the proposed approach, approach (compass), and traditional approach.

and thus the radio mismatch can be avoided effectively. For approach (compass), user orientation information is estimated by a compass and exploited in the fingerprinting pattern matching. However, the user orientation estimation accuracy is rather poor due to serve indoor magnetic perturbations. Therefore, the selected fingerprints may differ from the right fingerprints with similar orientations greatly. Therefore, the approach (compass) achieves small accuracy improvement than the traditional approach.

\section{Conclusions}

In this paper, we propose a novel WiFi fingerprinting positioning approach by considering both user orientations and carrying positions. Since the compass incurs large user orientation estimation errors due to indoor magnetic perturbations, we deploy RMPCA approach. For carrying position recognition, we propose a robust Random Forest classifier based on orientation invariant features, which may better adapt the changing device orientations. Under both LOS and NLOS environments, RSS patterns with different user orientations and smartphone carrying positions are distinct from one another. Therefore, the proposed WiFi fingerprinting approach may effectively avoid the radio mismatch during pattern matching. Experimental results show that compared with WiFi fingerprinting positioning approach only considering user orientation estimation using a compass and the approach considering neither user orientation nor carrying position information, our approach decreases mean positioning errors by $41.7 \%(1.15 \mathrm{~m})$ and $46.7 \%(1.41 \mathrm{~m})$, respectively. In our future works, we will further integrate pedestrian dead reckoning approach to improve the positioning performance.

\section{Conflicts of Interest}

The authors declare no conflicts of interest.

\section{Authors' Contributions}

Zhi-An Deng and Zhiyu Qu proposed the original idea and developed the proposed fingerprinting approach; Changbo Hou developed the user orientation algorithm; Weijian Si analyzed the data and revised the paper; Chunjie Zhang designed the experiments and gave some valuable suggestions.

\section{Acknowledgments}

This research is supported by the National Natural Science Foundation of China (Grant nos. 61671168, 61301132, 61300188, and 61301131) and the Fundamental Research Funds for the Central Universities (Grant nos. HEUCF180801 and HEUCFJ180801).

\section{References}

[1] Y. Sun, W. Meng, C. Li, N. Zhao, K. Zhao, and N. Zhang, "Human localization using multi-source heterogeneous data in indoor environments," IEEE Access, vol. 5, pp. 812-822, 2017.

[2] M. Cobos, F. Antonacci, A. Alexandridis, A. Mouchtaris, and B. Lee, "A survey of sound source localization methods in wireless acoustic sensor networks," Wireless Communications and Mobile Computing, vol. 2017, 2017.

[3] X.-L. Huang, J. Wu, W. Li, Z. Zhang, F. Zhu, and M. Wu, "Historical Spectrum Sensing Data Mining for Cognitive Radio Enabled Vehicular Ad-Hoc Networks," IEEE Transactions on Dependable and Secure Computing, vol. 13, no. 1, pp. 59-70, 2016.

[4] M. Zhou, Q. Zhang, K. Xu, Z. Tian, Y. Wang, and W. He, "PRIMAL: Page rank-based indoor mapping and localization using gene-sequenced unlabeled WLAN received signal strength," Sensors, vol. 15, no. 10, pp. 24791-24817, 2015.

[5] X. Huang, J. Wu, Y. Wen, F. Hu, Y. Wang, and T. Jiang, "Rate-Adaptive Feedback with Bayesian Compressive Sensing in Multiuser MIMO Beamforming Systems," IEEE Transactions on Wireless Communications, pp. 1-1. 
[6] X. Li, X. Zhang, X. Ren, M. Fritsche, J. Wickert, and H. Schuh, "Precise positioning with current multi-constellation Global Navigation Satellite Systems: GPS, GLONASS, Galileo and BeiDou," Scientific Reports, vol. 5, p. 8328, 2015.

[7] S. Gezici, Z. Tian, G. B. Giannakis et al., "Localization via ultrawideband radios: a look at positioning aspects of future sensor networks," IEEE Signal Processing Magazine, vol. 22, no. 4, pp. 70-84, 2005.

[8] J. Xiao, Z. Zhou, Y. Yi, and L. M. Ni, "A survey on wireless indoor localization from the device perspective," ACM Computing Surveys, vol. 49, no. 2, pp. 1-31, 2016.

[9] S. He and S.-H. G. Chan, "Wi-Fi fingerprint-based indoor positioning: recent advances and comparisons," IEEE Communications Surveys \& Tutorials, vol. 18, no. 1, pp. 466-490, 2016.

[10] G. Caso and L. De Nardis, "Virtual and Oriented WiFi Fingerprinting Indoor Positioning based on Multi-Wall Multi-Floor Propagation Models," Mobile Networks and Applications, vol. 22, no. 5, pp. 825-833, 2017.

[11] M. Zhou, F. Qiu, K. Xu, Z. Tian, and H. Wu, "Error bound analysis of indoor Wi-Fi location fingerprint based positioning for intelligent Access Point optimization via Fisher information," Computer Communications, vol. 86, pp. 57-74, 2016.

[12] L. Ma and Y. Xu, "Received signal strength recovery in a green WLAN indoor positioning system using singular value Thresholding," Sensors, vol. 15, no. 1, pp. 1292-1311, 2015.

[13] Z.-A. Deng, Y.-B. Xu, and L. Ma, "Indoor positioning via nonlinear discriminative feature extraction in wireless local area network," Computer Communications, vol. 35, no. 6, pp. 738-747, 2012.

[14] P. Bahl, V. Padmanabhan, and A. Balachandran, "Enhancements to the RADAR user location and tracking system," Microsoft Research, vol. 2, no. MSR-TR-2000-12, pp. 775-784, 2000.

[15] M. Youssef and A. Agrawala, "The Horus WLAN location determination system," in Proceedings of the 3rd International Conference on Mobile Systems, Applications, and Services (MobiSys '05), pp. 205-218, ACM, June 2005.

[16] S.-H. Fang and T.-N. Lin, "Indoor location system based on discriminant-adaptive neural network in IEEE 802.11 environments," IEEE Transactions on Neural Networks and Learning Systems, vol. 19, no. 11, pp. 1973-1978, 2008.

[17] Y. Xu, Z. Deng, L. Ma, W. Meng, and C. Li, "Signal perturbation based support vector regression for Wi-Fi positioning," in Proceedings of the 2012 IEEE Wireless Communications and Networking Conference, WCNC 2012, pp. 3123-3127, France, April 2012.

[18] S. Han, Z. Gong, W. Meng, C. Li, D. Zhang, and W. Tang, "Automatic Precision Control Positioning for Wireless Sensor Network," IEEE Sensors Journal, vol. 16, no. 7, pp. 2140-2150, 2016.

[19] L. Kanaris, A. Kokkinis, A. Liotta, and S. Stavrou, "Fusing bluetooth beacon data with Wi-Fi radiomaps for improved indoor localization," Sensors, vol. 17, no. 4, 2017.

[20] Z. A. Deng, Y. Bin Xu, and L. Ma, "Joint Access Point Selection and Local Discriminant Embedding for Energy Efficient and Accurate Wi-Fi Positioning," KSII Transactions on Internet and Information Systems, vol. 6, no. 3, pp. 794-814, 2012.

[21] K. Kaemarungsi and P. Krishnamurthy, "Properties of indoor received signal strength for WLAN location fingerprinting," in Proceedings of the 1st Annual International Conference on Mobile and Ubiquitous Systems: Networking and Services (MOBIQUITOUS '04), pp. 14-23, August 2004.
[22] S.-H. Fang, C.-H. Wang, and Y. Tsao, “Compensating for Orientation Mismatch in Robust Wi-Fi Localization Using Histogram Equalization," IEEE Transactions on Vehicular Technology, vol. 64, no. 11, pp. 5210-5220, 2015.

[23] Z. Zhang, X. Zhou, W. Zhang et al., "I am the antenna: accurate outdoor AP location using smartphones," in Proceedings of the 17th Annual International Conference on Mobile Computing and Networking (MobiCom '11), pp. 109-120, September 2011.

[24] F. Della Rosa, M. Pelosi, and J. Nurmi, "Human-Induced Effects on RSS Ranging Measurements for Cooperative Positioning," International Journal of Navigation and Observation, vol. 2012, Article ID 959140, 13 pages, 2012.

[25] F. Della Rosa, L. Xu, J. Nurmi, M. Pelosi, C. Laoudias, and A. Terrezza, "Hand-grip and body-loss impact on RSS measurements for localization of mass market devices," in Proceedings of the 2011 International Conference on Localization and GNSS, ICL-GNSS 2011, pp. 58-63, Finland, June 2011.

[26] T. King, S. Kopf, T. Haenselmann, C. Lubberger, and W. Effelsberg, "COMPASS: A probabilistic indoor positioning system based on 802.11 and digital compasses," in Proceedings of the WiNTECH 2006 - First ACM International Workshop on Wireless Network Testbeds, Experimental Evaluation and Characterization, pp. 34-40, USA, September 2006.

[27] D. Sánchez, J. M. Quinteiro, P. Hernández-Morera, and E. Martel-Jordán, "Using data mining and fingerprinting extension with device orientation information for WLAN efficient indoor location estimation," in Proceedings of the 2012 IEEE 8th International Conference on Wireless and Mobile Computing, Networking and Communications, WiMob 2012, pp. 77-83, Spain, October 2012.

[28] A. Papapostolou and H. Chaouchi, "Orientation-based radio map extensions for improving positioning system accuracy," in Proceedings of the International Wireless Communications and Mobile Computing Conference, pp. 947-951, ACM, June 2009.

[29] F. Ichikawa, J. Chipchase, and R. Grignani, "Where's the phone? A study of mobile phone location in public spaces," in Proceedings of the 2nd International Conference on Mobile Technology, Applications and Systems, pp. 1-8, IEEE, November 2005.

[30] E. M. Diaz, A. L. M. Gonzalez, and F. De Ponte Müller, "Standalone inertial pocket navigation system," in Proceedings of the 2014 IEEE/ION Position, Location and Navigation Symposium, PLANS 2014, pp. 241-251, USA, May 2014.

[31] Z.-A. Deng, G. Wang, Y. Hu, and D. Wu, "Heading estimation for indoor pedestrian navigation using a smartphone in the pocket," Sensors, vol. 15, no. 9, pp. 21518-21536, 2015.

[32] M. Kourogi and T. Kurata, "A method of pedestrian dead reckoning for smartphones using frequency domain analysis on patterns of acceleration and angular velocity," in Proceedings of the IEEE/ION Position, Location and Navigation Symposium (PLANS '14), vol. 19, pp. 164-168, IEEE, May 2014.

[33] L. Breiman, "Random forests," Machine Learning, vol. 45, no. 1, pp. 5-32, 2001.

[34] M. Shoaib, S. Bosch, O. Durmaz Incel, H. Scholten, and P. J. M. Havinga, "Fusion of smartphone motion sensors for physical activity recognition," Sensors, vol. 14, no. 6, pp. 10146-10176, 2014.

[35] Q. Tian, Z. Salcic, K. I.-K. Wang, and Y. Pan, "A MultiMode Dead Reckoning System for Pedestrian Tracking Using Smartphones," IEEE Sensors Journal, vol. 16, no. 7, pp. 20792093, 2016.

[36] Z. Deng, W. Si, Z. Qu, X. Liu, and Z. Na, "Heading estimation fusing inertial sensors and landmarks for indoor navigation 
using a smartphone in the pocket," EURASIP Journal on Wireless Communications and Networking, vol. 2017, no. 1, 2017.

[37] J. C. K. Chou, "Quaternion kinematic and dynamic differential equations," IEEE Transactions on Robotics and Automation, vol. 8, no. 1, pp. 53-64, 1992.

[38] Y. Li, J. Georgy, X. Niu, Q. Li, and N. El-Sheimy, "Autonomous calibration of MEMS gyros in consumer portable devices," IEEE Sensors Journal, vol. 15, no. 7, pp. 4062-4072, 2015.

[39] A. Brajdic and R. Harle, "Walk detection and step counting on unconstrained smartphones," in Proceedings of the ACM International Joint Conference on Pervasive and Ubiquitous Computing (UbiComp '13), pp. 225-234, September 2013.

[40] S. A. Hoseinitabatabaei, A. Gluhak, R. Tafazolli, and W. Headley, "Design, realization, and evaluation of uDirect-An approach for pervasive observation of user facing direction on mobile phones," IEEE Transactions on Mobile Computing, vol. 13, no. 9, pp. 1981-1994, 2014. 


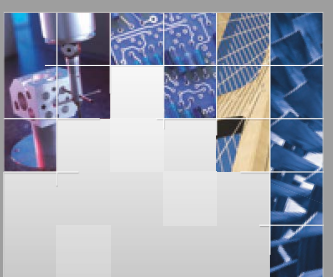

\section{Enfincering}
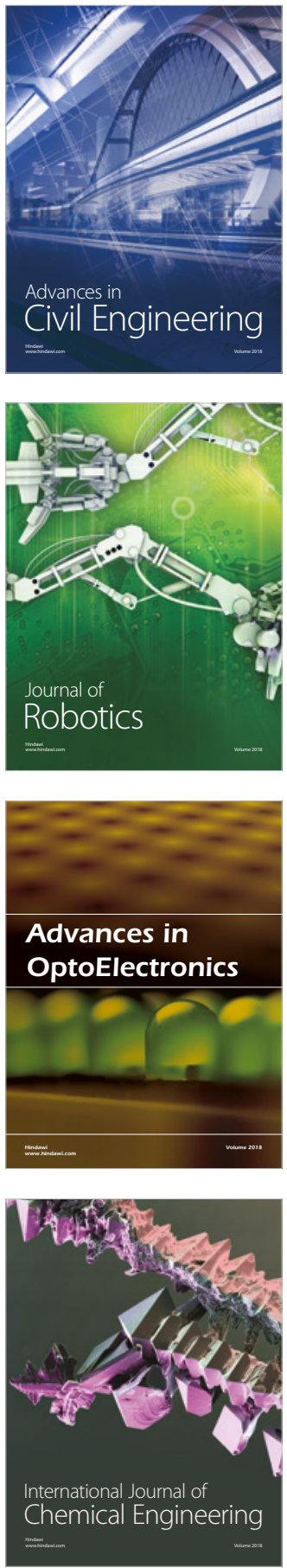

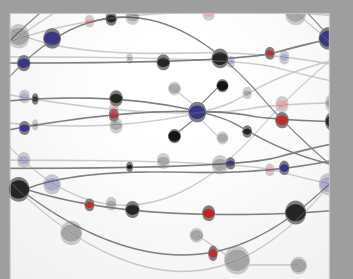

\section{Rotating \\ Machinery}

The Scientific World Journal

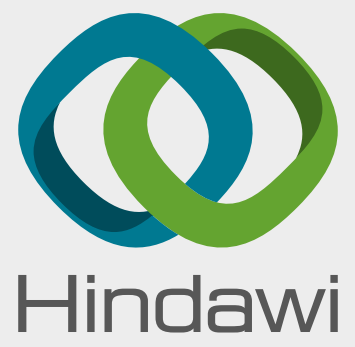

Submit your manuscripts at

www.hindawi.com
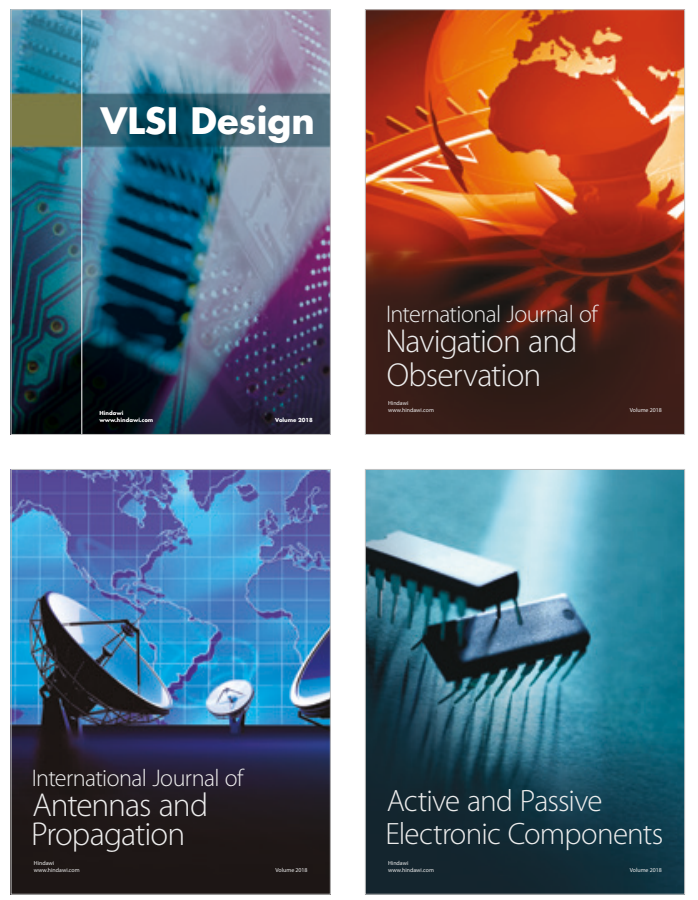
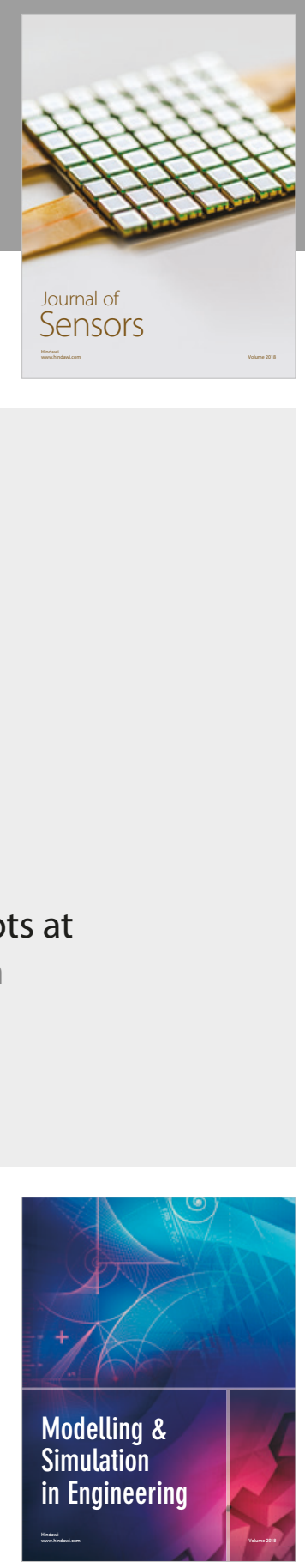

\section{Advances \\ Multimedia}
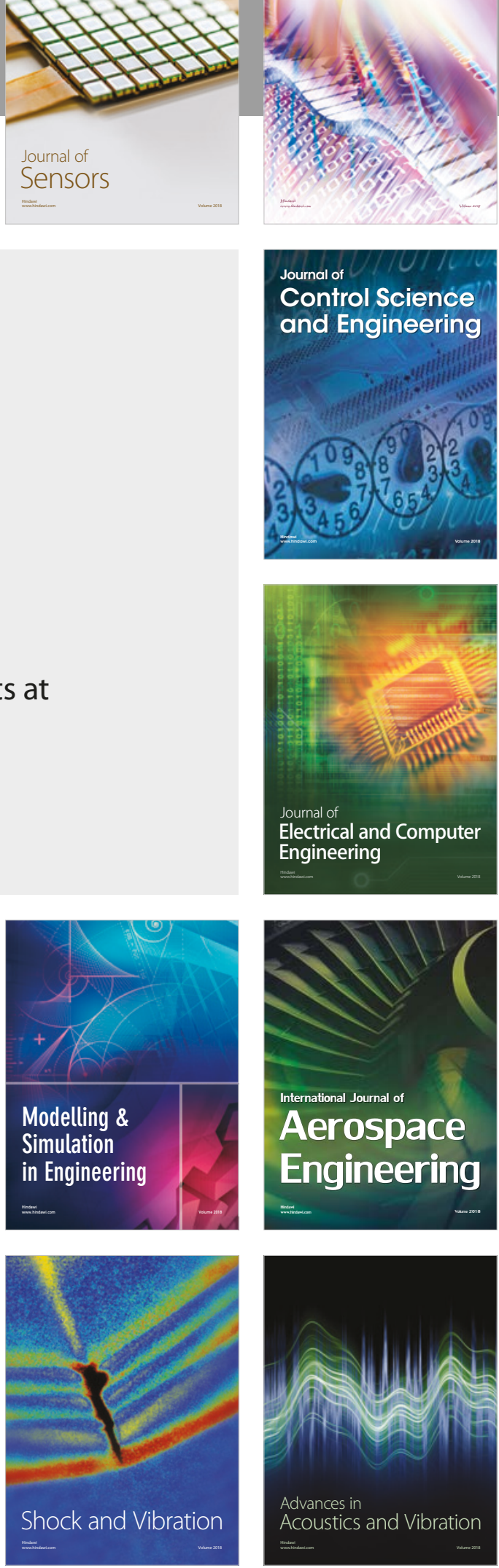